\title{
Space-Time Evolution of Subway Vulnerability Based on Hypergraph Space
}

\author{
Kaihua Lu $\mathbb{D}^{1,2}$ and Xiaoyan Gu $\mathbb{D D}^{3}$ \\ ${ }^{1}$ Nanjing University of Aeronautics and Astronautics, Nanjing 210000, China \\ ${ }^{2}$ Nanjing Public Engineering Construction Center, Nanjing 210000, China \\ ${ }^{3}$ Jinling Institute of Technology, Nanjing 210000, China \\ Correspondence should be addressed to Xiaoyan Gu; gxy@jit.edu.cn
}

Received 1 June 2020; Revised 18 September 2020; Accepted 27 September 2020; Published 14 October 2020

Academic Editor: Eric Florentin

Copyright (c) 2020 Kaihua Lu and Xiaoyan Gu. This is an open access article distributed under the Creative Commons Attribution License, which permits unrestricted use, distribution, and reproduction in any medium, provided the original work is properly cited.

\begin{abstract}
How to qualitatively and quantitatively evaluate the ability of the whole subway system to maintain the maximum normal operation has become a noteworthy topic. In this study, the hypergraph space structure of Nanjing subway network is used for research. This study uses the method of time-space evolution mechanism to dynamically and quantitatively analyze the gradual change characteristics of Nanjing subway system vulnerability under the influence of time and space. In addition, this study also makes static and dynamic comparative analysis on the vulnerability of a subway system by using connectivity rate and trip efficiency index, which provides new analysis methods and ideas for the study of a subway system network. The results show that although the overall structure of Nanjing's subway network shows a loose trend, as Nanjing's subway is still in a rapid development stage, it can be seen from the long-term planning of Nanjing's subway that the construction of Nanjing's subway ring line will be strengthened after the completion of the connection between the main city and the suburbs. Therefore, the vulnerability of the subway system should be reduced and the reliability will be improved.
\end{abstract}

\section{Introduction}

Subway, as an indispensable mode of transportation in urban transportation, is favored by people because of its safety, comfort, large carrying capacity, energy saving, environmental protection, convenience, and rapidity. However, with the busy operation of the urban subway, subway failures occur frequently, which are often directly manifested in a station or a section of the subway network that cannot work normally. According to statistics, the number of subway failures in Nanjing is about 1,000 a year [1]. Therefore, how to qualitatively and quantitatively evaluate the ability of the whole subway system to maintain the maximum normal operation has become a noteworthy topic.

In general network research, C. Pozrikidis presents a method to calculate spanning tree and uses binomial distribution to describe the node degree distribution of complete network spanning tree [2]. Gorjão et al. analyzed the complexity of network dynamics. After analysis, they obtained the influence of network size on dynamics research [3]. Alexander et al. deduced the complexity estimates of two types of deterministic networks. This breakthrough made it possible to create networks with the best complexity [4]. An et al. optimized the layout of traffic detectors using complex networks, which extended the practical application of complex networks in the field of transportation [5]. In addition, Karunakaran and Selvaganesh introduced the concept of neighborhood matrix. After that, the "neighborhood matrix" has become a new model that uses vertex neighborhood sets to represent graphs [6]. Beranek and Remes analyzed the intensity distribution and behavior characteristics of selected nodes and selected participants in the tripartite network, which further realized the systematic analysis of more complex tripartite networks [7]. Mensah et al. got rid of the limitations of small-scale networks and proposed an efficient and accurate shortest path algorithm 
for large-scale networks [8]. Džamić et al. introduced a new community structure detection standard for complex networks. This new detection standard provides a new path for community detection [9]. Latora and Marchiori proposed a complicated subway network model based on transportation efficiency [10]. Accordingly, Mrvar et al. studied the betweenness of the complex subway network [11]. These analyses of complex networks are often limited to general networks and cannot reflect the quantitative and other characteristics of subway networks.

With the development of social economy, various space structures have become increasingly complicated, so the analysis of indicators such as vulnerability of spatial models has also been cited in many aspects. Moreover, the vulnerability of complex networks was applied to the logistics service supply chain by $\mathrm{Ma}$ et al. [12]. Abdelaty et al. established a static robustness index by studying the robustness of the public transport network [13]. Polishchuk et al. introduced the concepts of parameter and betweenness. These two concepts can be used to determine the negative impact of complex network structure on internal and external [14]. Furthermore, Sun et al. considered the impact of passenger flow on the vulnerability of Shanghai's subway network [15]. Derrible and Kennedy established vulnerability evaluation indicators such as network redundancy and applied them to complex networks [16]. However, these analyses are only limited to the static analysis of network space and do not take into account the changes of dependent variables caused by the changes of time variables and space variables.

A dynamic model of transportation network evolution was proposed by Malinetskii and Stepantsov. At the same time, they also concluded that the dynamic behavior of the transportation network is the result of system self-organization [17]. Furthermore, Kim et al. proposed a fast time simulation method of air traffic network based on spacetime parallelism. Using this method, we can obtain greater acceleration in the traffic network [18]. It is worth noting that Hosseini and Talebpour used space-time diagrams to obtain better prediction results [19]. On this basis, Su et al. applied time-space correlation analysis to road traffic congestion [20]. Bačkalić et al. found the coordinates of the traffic accident by the space-time coordinate system. More specifically, they proposed a new method to study the time interval of traffic accidents [21].

Additionally, $\mathrm{Qu}$ and $\mathrm{He}$ used the spatiotemporal network model to predict and control station traffic congestion caused by emergencies. At the same time, they also verified the feasibility of the model [22]. Kang et al. conducted many long-term studies on the mechanism of how the temporal and spatial characteristics of traffic accidents for the elderly in Seoul change over time [23]. Based on the research of the predecessors, some new methods were born as follows. Specifically, Zawack and Thompson developed a spatiotemporal network to represent the traffic flow of the road traffic system over time [24]. On the basis of considering only points or lines in the past, Sun and Guan proposed to measure the vulnerability of the subway network from the perspective of line operation [25]. Ding et al. established a short turn model. And through this model, they strived to alleviate the current situation of overcrowding in dense traffic demand effective methods [26]. Nian et al. proposed a quantitative evaluation method. This method can be used to evaluate the performance of urban subway networks under various interference conditions [27]. Crucitti et al. made use of the network efficiency concept to study scale-free networks, and the conclusion which global efficiency can better reflect the response of complex networks to external factors than characteristic path lengths can be drawn [28]. Based on traffic flow redistribution, Su et al. proposed a cascading failure model and applied it to the transportation network studies [29].

With the acceleration of China's urbanization process, the pressure brought by the increase of urban population on urban traffic has become increasingly obvious. However, the speed of urbanization must not be restricted by traffic pressure. Therefore, underground subway traffic, which corresponds to the traditional aboveground traffic, has become a new channel to relieve the traffic pressure in cities. In the future, 43 cities in China will build subways. It can be said that China is in a period of rapid development of subway. Moreover, with the continuous increase of new subway lines, the overall transportation network of the Nanjing subway system will be gradually improved.

\section{Hypergraph Space of Nanjing Subway}

Claude Berge, a French mathematician known as the father of modern graph theory, expanded the edges of graphs to superedges. The expansion of this small step has triggered the development of a large field.

The edges of hypergraphs are defined as superedges, which can be connected with any number of vertices.

Suppose $V=\{v 1, v 2, v 3, \ldots, v n\}$ is a finite set and $E=\{e 1, e 2, e 3, \ldots, e m\}$ is a set family of a series of subsets of $v$.

If $e i \neq \varnothing(i=1,2, \ldots, m)$ and $\cup e i=V(i=1,2, \ldots, m)$, then set family $H=(V: e 1, e 2, e 3, \ldots, e m)$ is considered to be a hypergraph of finite set $V$. Among them, $U e i$ is the union of $e 1, e 2, e 3, \ldots, e m$.

Elements $v 1, v 2, v 3, \ldots, v n$ are called vertices of hypergraphs, while sets $e 1, e 2, e 3, \ldots, e m$ are called superedges of hypergraphs. Each superedge is a subset of $V$, and $E=\{e 1, e 2$, $e 3, \ldots, e m\}$ is the set of hyperedges and superedges.

\subsection{Matrix Representation of Hypergraphs}

$$
H(V, E)= \begin{cases}1, & v i \in e j, \\ 0, & 0, v i \notin e j .\end{cases}
$$

If $i=5, j=4$; the hypergraph matrix is

$\begin{array}{ccccc} & e_{1} & e_{2} & e_{3} & e_{4} \\ v_{1} & 1 & 0 & 0 & 0 \\ v_{2} & 1 & 1 & 1 & 1 \\ v_{3} & 0 & 0 & 1 & 1 \\ v_{4} & 0 & 1 & 0 & 0 \\ v_{5} & 0 & 0 & 0 & 0\end{array}$.

The corresponding hypergraph is 


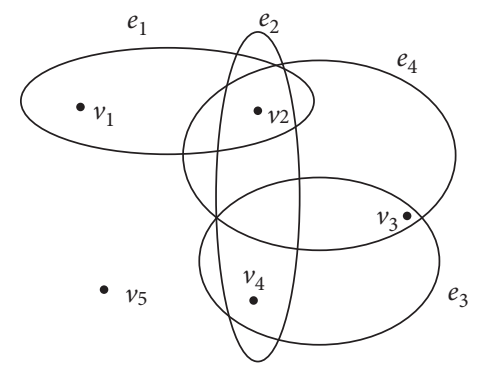

$V=\{v 1, v 2, v 3, v 4, v 5\}$,

$E=\{e 1, e 2, e 3, e 4\}=\{\{v 1, v 2\},\{v 2, v 4\},\{v 3, v 4\},\{v 2, v 3\}\}$.

The hypergraph matrix space is

$$
\mathrm{B}_{m \times n}=v_{3} v_{1}\left[\begin{array}{cccccc}
v_{1} & e_{2} & e_{3} & e_{4} & \ldots & e_{n} \\
v_{4} & : \\
b_{11} & b_{12} & b_{13} & b_{14} & \ldots & b_{1 n} \\
b_{i 1} & b_{22} & b_{23} & b_{24} & \ldots & b_{2 n} \\
b_{31} & b_{32} & b_{33} & b_{34} & \ldots & b_{3 n} \\
b_{41} & b_{42} & b_{43} & b_{44} & \ldots & b_{4 n} \\
: & : & : & : & \ldots & : \\
b_{m 1} & b_{m 2} & b_{m 3} & b_{m 4} & \ldots & b_{m n}
\end{array}\right] .
$$

$e i(i=1,2,3, \ldots, n)$ is the no. $i$ operation line. $v_{i}(i=1,2$, $3, \ldots, n)$ is the no. $i$ transfer station. $n$ is the number of lines currently in operation. $m$ is the number of transfer stations and terminal stations currently in operation. $b_{i j}(i=1,2,3$, $\ldots, n)$ is a representation of the correlation between lines.

$B_{n \times n}=\left(e_{n}, e_{n}\right)$ is a noncontiguous matrix ( $n$ is not equal to $m$ ), which reflects the relationship between each operation line and all transfer stations or terminal stations. When the transfer station or terminal station is on this operation line, $b_{i j}$ takes 1 ; otherwise, it takes 0 .

In network space and space $P$, there are certain limitations in the expression of stations and lines. Only when there are connections or intersections between stations or lines can there be bordering. However, the simple connection between the two points can only be reflected by a simple connection, which cannot well express the relationship between the subsystem and the overall system. The advantage of hypergraph space structure is that the connection of hyperedges is not affected by feature sets, and it can be described more by using its features; that is, the point sets with different features can better reflect their correlation characteristics. At the same time, hypergraph space structure can not only describe the finite set freely but also segment the data and feature points ideally and vividly, which can well reflect the relationship and position of vertices in the finite set in the whole. Using hypergraph space structure is also one of the differences between this study and other studies.

\section{Date Collation}

First of all, the overall construction plan of Nanjing subway fully meets the travel conditions of Nanjing's urban population. Secondly, the overall construction plan of Nanjing subway is compatible with the city's economic development. In 2018, Nanjing's subway had a total of 1118.888 million passengers, with an average daily passenger volume of 3.07 million. We can clearly see that more and more people take the subway as their preferred means of transportation. Therefore, the ability of the city subway system to maintain normal operation has become one of the important indicators to evaluate the quality of a city's public utilities.

According to official website of Nanjing Metro, by the end of 2019, Nanjing has successively opened lines 1, 2, 10, S1, S8, 3, 4, S3, S9, and S7, totaling 10 operating lines.

From Figure 1, it can be seen that Nanjing's subway forms the entire subway system space network through various lines distributed in the longitude and latitude directions, and shows a trend of extending outward. Among them, lines 2, 4, and 10 run from east to west, while lines 1 and 3 run from north to south, forming the subway network of Nanjing's main city. Lines S3, S1, S7, S8, and S9 are lines connecting the main city to the suburbs, showing a divergent trend in all directions.

Moreover, as can be seen from Figure 1, Nanjing subway stations generally form commercial centers and transportation hubs together with railway stations, bus stations, and airports, while other modes of transportation form a star-shaped space structure of transportation network around these important subway stations.

With the opening and operation of new subway lines, Nanjing subway gradually formed a complex network, which also created conditions for the study of Nanjing subway as a whole as a network space structure.

\section{Network Space and $P$ Space of Nanjing Subway}

In Nanjing's subway network space, if transfer stations or terminal stations are connected in pairs, there will be a bordering between the two stations, and if subway operation lines can be transferred through stations, there will be a bordering between the two lines, thus establishing Nanjing's subway network space and space $P$ structure. Considering the difference between the vertical operation and departure frequency of Nanjing subway lines, the author sets the network space of Nanjing subway as an unweighted and undirected network structure.

Figure 2 shows that there are 10 nodes in the space structure of Nanjing subway network, i.e., 10 subway lines (until the end of 2019). In network space, there are no isolated nodes. This shows that all nodes are reachable, which means that all subway lines can reach each other through transfer stations.

As can be seen from Figure 3, in Nanjing's subway space $P$ structure, there are 26 nodes, that is, 26 transfer stations and terminal stations. We can also see that in the space $P$ structure of Nanjing subway, there is a large spacing between the nodes, which is consistent with the characteristics of the larger overall area of Nanjing city and sparse distribution of stations at subway transfer stations or terminals.

As can be seen from Figures 2 and 3, Nanjing's urban subway network shows two characteristics: ring structure 


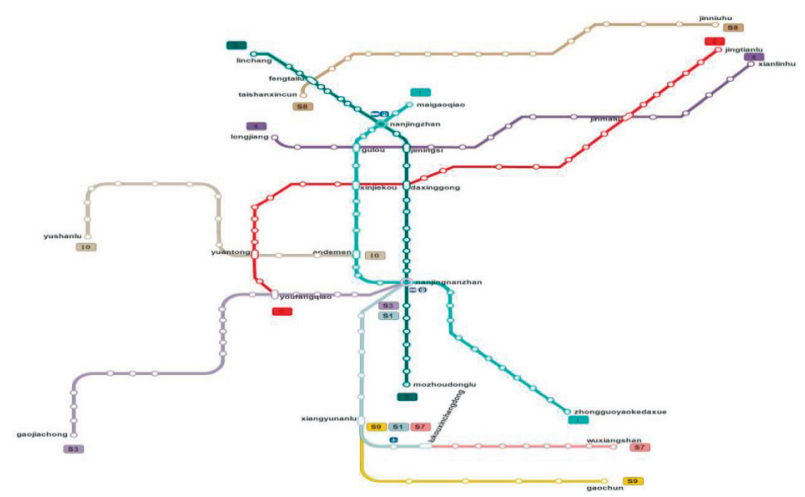

Figure 1: L-shaped space topological diagram of the Nanjing subway.

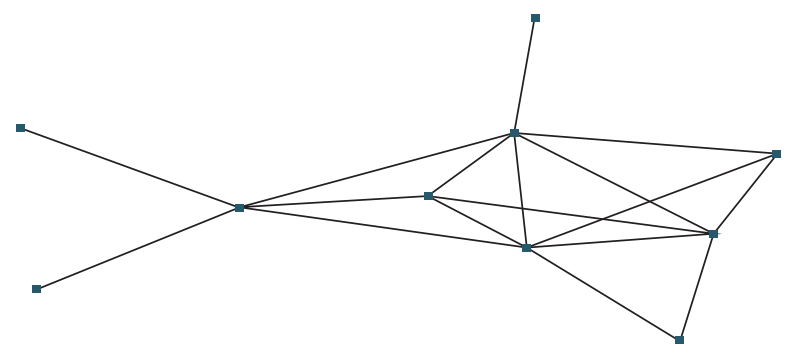

FIGURE 2: Network space.

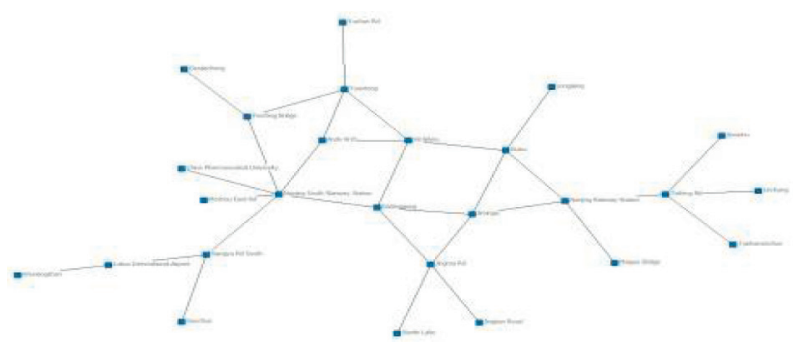

Figure 3: Space $P$.

and star structure. In the center of the subway network, a ring-shaped structure is formed and radiates to the surrounding remote areas, which is in line with the plan of constructing the main city line and the suburban line to realize the full coverage of subway traffic in the initial stage of urban subway construction.

In the network space model, the sum of the connection times of all the connected edges with node $v i$ is the node degree of node $v i$. When a node has no associated edge with other nodes in the network, the node degree is $0 . P(k)$ represents the distribution probability of all nodes with node degree $k$, and the sum of $P(k)$ values of all nodes is 1 .

In Nanjing's subway network space, lines 1 and 3 have the largest node degree, with a node degree of 6 . Lines S7, S8, and $\mathrm{S} 9$ have the smallest node degree, and the node degree is 1. Lines 1 and 3 are most closely connected to other lines and have the greatest connectivity in the subway network, while lines S7, S8, and S9 are in line with their transportation functions, which mainly connect the main cities and suburbs.
In the space $P$ of Nanjing subway, the Nanjing South Railway Station has the largest node degree of 6, while 14 stations such as Yushan Road have the smallest node degree of 1 . In the whole subway space $p$, the station with the highest node degree has the most connections with the surrounding stations. Judging from the time sequence of subway construction, the later-stage construction lines are generally connected to the already-operated lines, which also leads to more connections between the stations of the already-operated lines and the later-stage construction stations. Judging from the time sequence of subway construction, the later-stage construction lines are generally connected to the already-operated lines, which also leads to more bordering between the stations of the already-operated lines and the later-stage construction stations.

From Figure 4, we can see that the node degree distribution of the whole network is seriously uneven. At the same time, it also shows the serious heterogeneity and scale-free of the subway network [30].

\section{Basic Measure of Hypergraph Space}

5.1. Node Superdegree. In hypergraph space, the sum of the number of all nodes connected to node $v$ through superedges is the node superdegree of node $v$. In Nanjing subway hypergraph space structure, the hypergraph nodes are composed of transfer stations and terminal stations.

Figure 5 shows the top 10 stations for node overcrossing. Among them, the Nanjing South Railway Station has the highest node superdegree. The station has the closest connection with other stations, and the other stations that transfer from this station are also the most. The Gaochun Station and Wuxiangshan Station have the smallest node superdegree, because both stations are at the end of the line and have the weakest connection with other stations.

As can be seen from Figures 4 and 6 , in the subway network space of Nanjing, the maximum value of $P(k)$ is 0.3 and the minimum value is 0.1 . In Nanjing subway space $p$, the maximum value of $P(k)$ is 0.16 and the minimum value is 0.01 . In the network space and $P$ space structure, the proportion of node degree 1 is relatively large. In hypergraph space structure, the maximum value of $P(k)$ is 0.15 and the minimum value is 0.04 . Compared with network space structure and space $P$ structure, the degree distribution of nodes in hypergraph space structure is more uneven.

The number of nodes and borderings represents the scale of the subway network. Nanjing's subway network space, space $P$, and hypergraph space structure are of the same size. As new subway lines and stations are put into operation, the size of Nanjing subway space will also increase, indicating that the size of subway space is linearly related to the number of subway lines and stations.

5.2. Superedge Degree. In hypergraph space structure, the sum of the number of superedges associated with superedge $e$ is the hyperedge degree of superedge $e$, which reflects the closeness of the superedge $e$ to other superedges. In the subway hypergraph space, the operation line is superedge. 


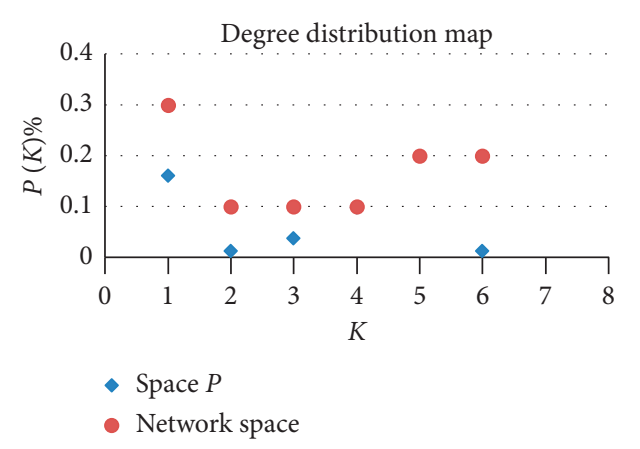

Figure 4: Probability diagrams of node degree distribution (network space, space $P$ ).

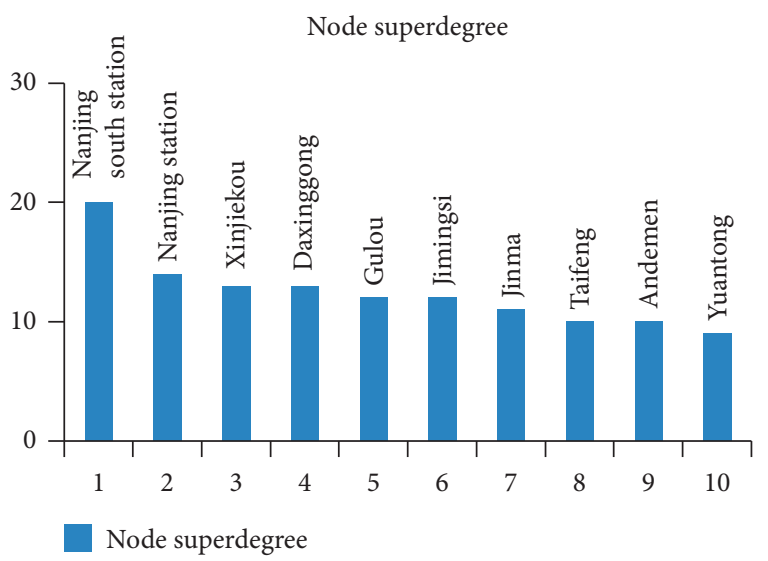

FIGURE 5: Ranking of node superdegree in the Nanjing subway station.

Figure 7 is an operation line arranged from large to small according to the size of superedge of hypergraph space. The average superedge degree of Nanjing subway is 3.4, of which, lines 1 and 3 have the largest superedge degree, and these two lines run south to north across the commercial circle of Nanjing's main urban area. At the same time, because these two lines operate earlier and thus have more transfer nodes with other lines, they are also most accessible to other lines.

Among them, lines S7, S8, and S9 have the smallest superedge degree. This is because the three lines are mainly to realize the function of suburban personnel to reach the main city, and the connection between the routes to the suburbs and the main city is relatively weak. According to the ranking and superedge degree, the line after the fifth place basically only serves the function of connecting the main city and the suburbs.

5.3. Superedge Weight. In subway hypergraph space, superedge weight refers to the number of transfer stations and terminal stations included in the line, which reflects the parameter of the connection function of the superedge in hypergraph space structure. If there are more transfer stations and terminal stations in the line, then the value of superedge weight will be larger, which indicates that the line plays a more important role in the overall subway system.

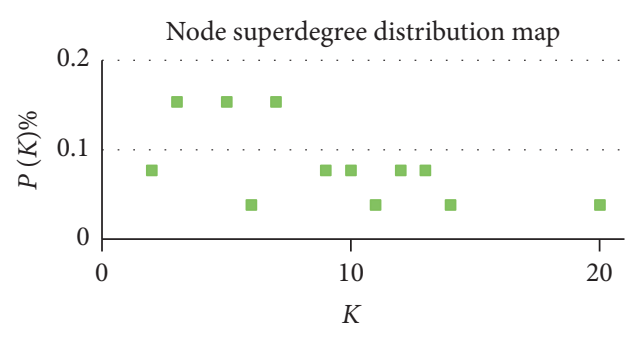

- Hypergraph

Figure 6: Probability diagrams of node degree distribution (hypergraph space).

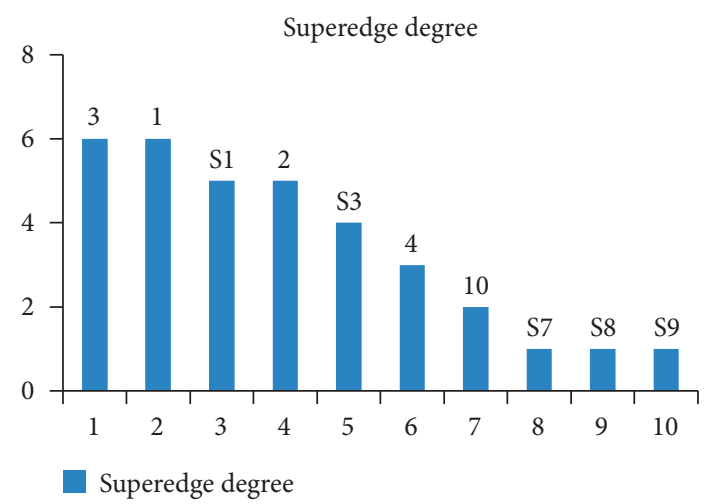

FIGURE 7: Ranking of superedge degree of Nanjing subway.

Figure 8 shows an operation line arranged from large to small according to the size of superedge weight in hypergraph space. In Nanjing subway hypergraph spatial structure, lines 1 and 3 have the largest superedge weight, while lines S7 and S9 have the smallest superedge weight, with an average superedge weight of 4.1. The sorting results calculated by superedge weight are basically consistent with the sorting results calculated by superedge degree.

In the space structure of the Nanjing subway network, the average node degree is 3.4 , the clustering coefficient is 0.676 , and the network centrality is $36.11 \%$. In the spatial $P$ structure of Nanjing subway, the average node degree is 2.4, the clustering coefficient is 0.128 , and the network centrality is $15.67 \%$. In Nanjing subway hypergraph space structure, the average node hyperdegree is 7.8. In hypergraph space structure, the nodes are most closely connected, but the center aggregation degree of subway network space is the highest.

\section{Definition and Indicators of Subway System Vulnerability}

The vulnerability of the subway system refers to the characteristic that some performance of the subway system will change when some parameters change cumulatively in the subway system. Vulnerability is one of the main dynamic performances of the subway system, which reflects the stability and anti-interference of the subway system.

Specifically, subway system vulnerability refers to the degree of change of certain functions of the new subway 


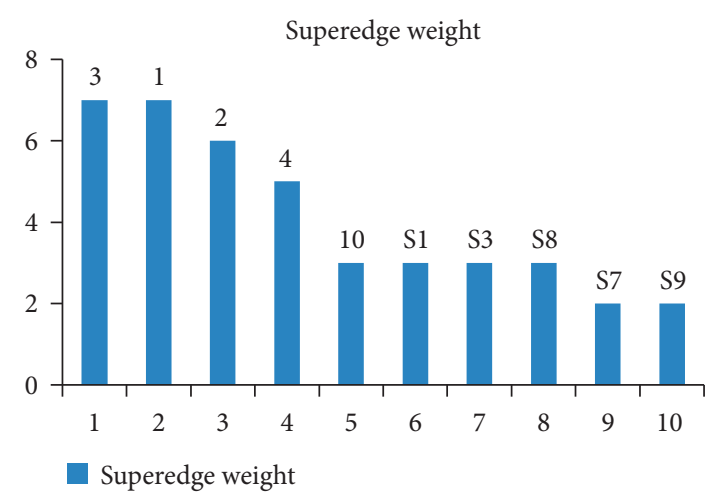

FIgURE 8: Superedge weight ranking of Nanjing subway.

system when important operating lines or key stations lose their functions. This paper mainly analyses the manifestation of Nanjing subway system vulnerability in the hypergraph spatial structure.

6.1. Connectivity Rate. Connectivity rate $f$ is a dynamic indicator reflecting the vulnerability of the subway system, which is generally less than 1 . The greater the connectivity rate, the higher the connectivity efficiency of the new subway system, and the faster the arrival of each node. Connectivity change rate is an indicator to quantitatively display the degree of connectivity change:

$$
f=\frac{X}{\sum_{i=1}^{N} \sum_{j=1}^{N} l(v i, v j)(i \neq j)} .
$$

Among them, $N$ represents the number of stations; $l(v i, v j)$ is the distance from node $v i$ to node $v j . l(v i, v j)$ is also the minimum number of connected edges from node $v i$ to node $v j ; X$ is the number of sides that all stations are connected to each other.

6.2. Trip Efficiency. In the subway system, when there are multiple connection paths between two stations, most passengers will choose the shortest route, so the trip efficiency $Q$ is defined as the average of the reciprocal of the shortest path between all two stations in the hypergraph space of the whole subway system. Trip efficiency is a measurement index to measure the efficiency change of the subway system after certain parameters change:

$$
Q=\frac{1}{N(N-1)} \sum_{i=1}^{N} \frac{1}{l(v i, v j)} \quad(i \neq j) .
$$

In the formula, $N$ is the number of stations. $l(v i, v j)$ represents the distance between node $v i$ and node $v j$.

\section{Vulnerability Analysis of Nanjing Subway System (Static Characteristics)}

Vulnerability analysis is generally verified by random attacks or deliberate attacks on stations and lines in subway systems. However, considering that deliberate attacks are more destructive than random attacks, this paper only analyses the vulnerability of the Nanjing subway system through deliberate attacks in the most unfavorable conditions.

7.1. Attacks on Nanjing Subway Stations according to the Order of Node Superdegree. According to Figure 5, the order of attacking stations is determined according to the order of node superdegree in the hypergraph space model of Nanjing subway

In Figure 9, the abscissa represents the station order number from large to small according to node superdegree. Abscissa 1 is the initial state of subway network ( $Q$ is 0.28 , and $f$ is 0.027). Abscissa 2 represents the Nanjing South Railway Station. Abscissa 5 represents the Daxinggong Station. Abscissa 11 represents the Yuantong Station.

According to Figure 9, when the station is attacked in Nanjing subway hypergraph space, the Daxinggong Station located in the city center has the greatest impact on the vulnerability of Nanjing subway ( $Q$ increased from 0.34 to 0.54 , with a change rate of $60 \%$ ). This station is the transfer station of line 1 and line 3 with the highest superedge degree and usually has a large daily passenger flow, so it is also the most disturbing in the whole Nanjing subway system. When Gulou Station, Jiming Temple Station, and Jinma Road Station were attacked, the impact on the overall function of the Nanjing subway system was relatively weak, which was consistent with the line characteristics of the three stations that were not busy. The traffic volume of these three stations is also small at ordinary times. $Q$ and $f$ present a downward trend in general given the context of the sequential failures of subway stations. There is a negative correlation between the changes in travel efficiency and changes in network vulnerability. The subway network has lost its basic operational functions after 18 stations were under attack in the subway network system.

7.2. Attacks on Nanjing Subway Lines according to the Ranking of Superedge Degree. From Figure 7, the superedge degree in the Nanjing subway hypergraph space model is sorted to determine the order of attack routes.

In Figure 10, the abscissa 1 is the initial state of subway network $(Q$ is $0.55, f$ is 0.21$)$. Abscissa 2 represents line 3 . Abscissa 4 represents line S1.

From Figure 10, we can see that in the hypergraph space of Nanjing subway, line S1 has the greatest impact on the vulnerability of Nanjing subway when there are lines under attack. Line S1 is the Nanjing South Railway Station with the highest node overcrossing and the suburban line, and it is also responsible for connecting S7 and S9 suburban lines with the overall subway system. When lines 1,3 , and S1 were attacked, the remaining lines of the Nanjing subway system were isolated and could not form an overall hypergraph spatial structure.

7.3. Attack Nanjing Subway Line according to Superedge Weight. From Figure 11, it can be seen that in Nanjing subway hypergraph space, when the line is attacked, line 1 has the greatest impact on the vulnerability of Nanjing subway. Line 1 is the line connecting the Nanjing South 


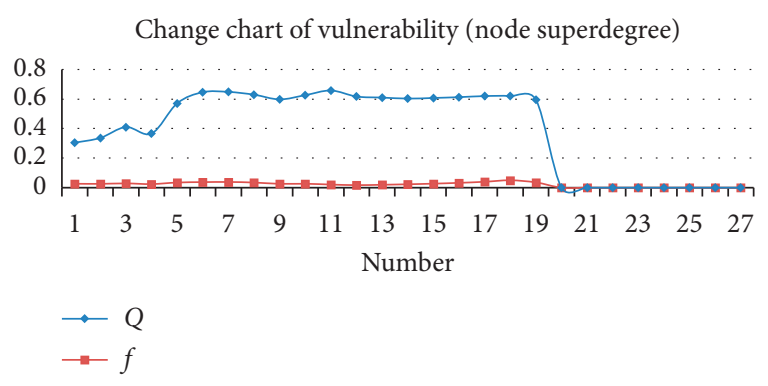

Figure 9: Vulnerability change of Nanjing subway (node superdegree).
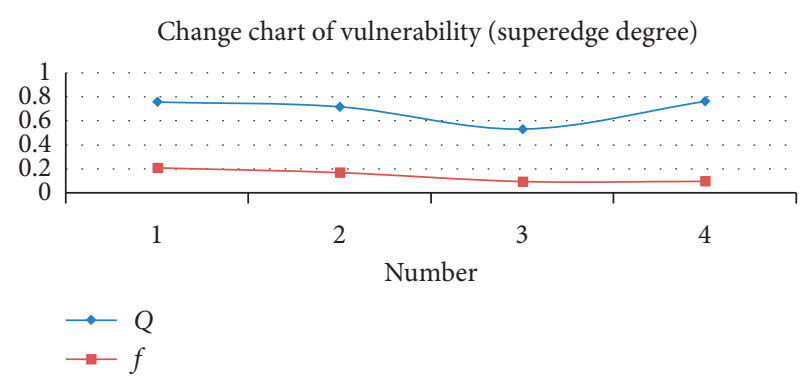

FIGURE 10: Vulnerability change of Nanjing subway (superedge degree).

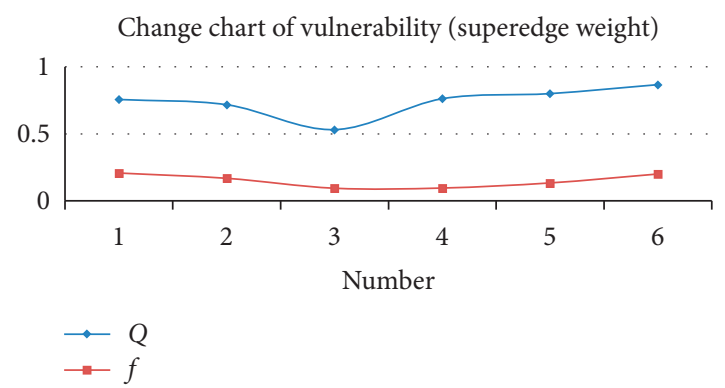

FIGURE 11: Vulnerability change of Nanjing subway (superedge weight).

Railway Station and Nanjing Station with the highest node overcrossing, and it passes through the Xinjiekou Station with the largest daily passenger flow. When lines $1,2,3,4$, and 10 were attacked, the Nanjing subway system lost its operation function. Compared with the superedge degree attack, the Nanjing subway system will be affected relatively slowly according to the superedge weight attack.

Compared with public transportation lines, subway lines generally do not overlap. When a major subway line or station fails, the entire subway network will be obviously divided into isolated subsystems, resulting in a rapid decline in network connection efficiency. Therefore, the overall improvement strategy to improve the vulnerability of the subway system is the key node to strengthen the vulnerability index, and the tightness and connectivity between the relatively independent subsystem structure and the subway main system should be correspondingly increased.

\section{Space-Time Evolution of Nanjing Subway Vulnerability (Dynamic Characteristics)}

In Table 1, line 10 and line S1 are basically operated simultaneously. Among them, subway line 10 started trial operation on February 26, 2014, and line S1 started trial operation on March 10, 2014. The opening and operation time of subway line 10 should be set earlier than that of line S1. Table 1 shows the sequence of opening of each line in Nanjing subway transportation network, as well as important transportation network nodes such as transfer stations and terminal stations on the line. We can see that the subway lines built in the later period will be connected to the existing lines through transfer stations as much as possible and gradually form a new subway traffic network with close ties.

Referring to Figure 1 and Table 1, with the opening of metro lines, the spatial structure of Nanjing Metro network has changed from unilateral spatial structure formed by line 1 to multilateral complex spatial structure by the end of 2019.

\subsection{Space-Time Evolution of Nanjing Subway Connectivity} Rate $(f)$. According to the time sequence of line construction in Table 1, we can obtain the space-time evolution characteristics of hypergraph spatial connectivity rate of Nanjing subway (Figure 12), with an average connectivity of 0.48 . The maximum connectivity rate of 1 appears after the opening and operation of lines 2 and 10, while the minimum connectivity rate of 0.2 appears after the opening and operation of line S7.

Connectivity rate shows the accessibility between subway lines. With the increase in the number of subway lines and stations, the overall connectivity rate will show a downward trend, at which time the accessibility between subway lines will decrease. After the completion and opening of lines $\mathrm{S} 1$ and S8, the connectivity rate has reached a turning point of decline, because line $S 1$ is the fourth completed and operated line of Nanjing subway, which mainly undertakes the transportation function between the Lukou International Airport Station and the main urban area. Line S8 is the fifth subway line built and operated in Nanjing and is also the first subway line in Nanjing with its entire length located north of the Yangtze River. Before the completion of line S8, all the subway lines in Nanjing were located south of the Yangtze River and had formed a subway transportation network in Nanjing's main urban area. The completion and operation of line S8 is a single-track independent operation, which is not connected with the subway transportation network in the main urban area south of the Yangtze River. Subsequently, line S8 and the subway network in the main urban area are connected and communicated with each other from line 3 , which also leads to the increase of the connectivity rate from 0.3 to 0.34 after the completion and operation of line 3. After that, the connectivity rate remained basically stable. We can see that the connectivity rate is directly proportional to the accessibility of the subway. At the same time, the subway 
TABle 1: Transfer station and terminal station of Nanjing subway line.

\begin{tabular}{lccc}
\hline Line & $\begin{array}{c}\text { Number of } \\
\text { stations }\end{array}$ & Transfer station and terminal station & Opening time \\
\hline 1 & 27 & China Pharmaceutical University, Nanjing South Railway Station, Andemen, Xinjiekou, Gulou, & Nanjing Railway Station, Maigaoqiao \\
2 & 26 & Youfangqiao, Yuantong, Xinjiekou, Daxinggong, Jinmalu, Jingtianlu & 2010.5 .28 \\
Andemen, Yuantong, Yushanlu Station & 2014.7 .1 \\
10 & 14 & Lukou International Airport Station, Xiangyulunan Station, Nanjing South Railway Station & 2014.7 .1 \\
S1 & 9 & Jinniuhu Station, Taifenglu, Taishanxincun Station & 2014.8 .1 \\
S8 & 17 & Taifenglu, Mozhoudonglu Station, Nanjing South Railway Station, Daxinggong, Jimingsi, Nanjing & 2015.4 .1 \\
3 & 29 & Railway Station, Linchang Station & 2017.1 .18 \\
4 & 18 & Xianlinhu, Jinmalu, Jimingsi, Gulou, Longjiang & 2017.12 .6 \\
S3 & 19 & Nanjing South Railway Station, Youfangqiao, Gaojiachong & 2017.12 .30 \\
S9 & 6 & Xiangyulunan Station, Gaochun Station & 2018.5 .26 \\
S7 & 9 & Wuxiangshan Station, Lukou International Airport Station & \\
\hline
\end{tabular}

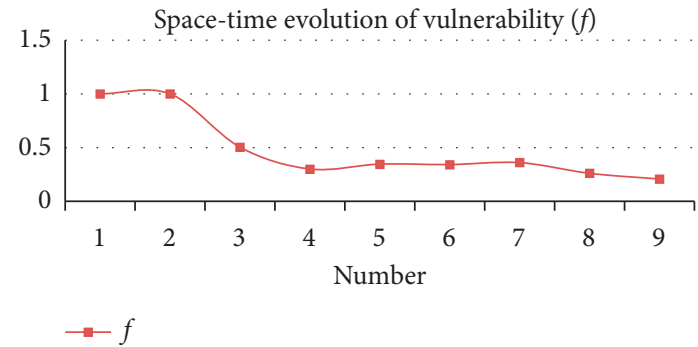

FIGURE 12: Space-time evolution of vulnerability $(f)$.

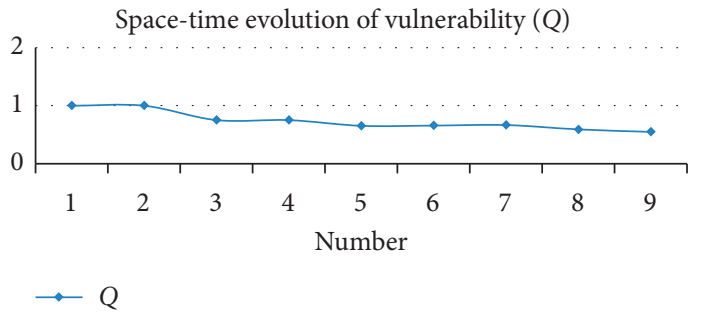

FIGURE 13: Space-time evolution of vulnerability $(Q)$. line plays a major role in the whole public transportation network, which is different from the trunk transportation of public buses and other short-distance transportation means.

8.2. Space-Time Evolution of Nanjing Subway Trip Efficiency (Q). According to the time sequence of line construction in Table 1, we can obtain the space-time evolution characteristics of Nanjing subway hypergraph space trip efficiency (Figure 13), with an average trip efficiency of 0.74 . When lines 2 and 10 are put into operation and connected with line 1 , the maximum trip efficiency is 1 , while the minimum trip efficiency is 0.55 after line S7 is put into operation. At the same time, the opening of the new subway lines has not only expanded the scale of the network but also loosened the overall network.

Trip efficiency can reflect the convenience of trip. However, the overall trip efficiency in Nanjing shows a downward trend. After the completion of line S1, the trip efficiency showed a significant turning point of decline and then remained basically stable. After the completion and opening of line S3, trip efficiency will be improved. Line S3 passes through the Nanjing South Railway Station and Youfangqiao Station, and connects lines 1, 2, 3, and $\mathrm{S} 1$, indirectly playing the role of a loop, which also leads to the closure of subway operation lines in Nanjing's downtown business circle. This is the reason why the completion and opening of S3 line leads to the improvement of efficiency. Line S9 and S7 are subway lines connecting the main city and the suburbs. There are few connections with the main city subway network. When these two lines are completed and opened, the trip efficiency will drop to the lowest level.

At present, the overall trip efficiency of Nanjing's subway shows a downward trend, because the number of lines currently opened by Nanjing's subway is relatively small, and the newly opened lines are still mainly responsible for transportation in the main city and suburbs, without adding subway loops, which also leads to the loose connection between the entire Nanjing subway lines. However, it can be seen from the long-term planning of Nanjing subway that the construction of the Nanjing subway ring line will be increased after the completion of the connecting line between the main city and the suburbs, which will lead to the improvement of trip efficiency.

The static characteristics and space-time evolution characteristics of Nanjing subway vulnerability are compared and analyzed. Static characteristic analysis is used to analyze the vulnerability of the subway network after the Nanjing subway line is completed and operated and a relatively fixed and balanced network space is formed. This static state may be a short-term equilibrium or a long-term equilibrium. On the contrary, space-time evolution analysis is a vulnerability analysis that changes the independent variables and dependent variables accordingly. Compared with space-time evolution analysis, static analysis has the biggest difference that it does not consider the changes of results brought by the changes of time and space variables. Therefore, we can see that the static analysis of Nanjing subway vulnerability focuses on strengthening and focusing on the weak nodes of 
the established subway network, while the space-time evolution analysis of Nanjing subway focuses on the forwardlooking planning of weak nodes caused by time and space factors, which is also the difference between this study and previous studies.

\section{Conclusion}

Subway, as an indispensable mode of transportation in urban transportation, has increasingly shown its important position in the development of urban transportation pattern due to its advantages of convenient travel and the local economic development driven by it. However, with the busy operation of the urban subway, subway failures occur frequently, which are often directly manifested in a station or a section of the subway network that cannot work normally. Therefore, how to qualitatively and quantitatively evaluate the ability of the whole subway system to maintain the maximum normal operation has become a noteworthy topic. By the end of 2019, Nanjing subway has already opened and operated 10 subway lines, basically forming a complex network spatial structure and possessing the space-time evolution characteristics of the space structure.

In the past, scholars usually used the general network to analyze the subway, but this kind of analysis cannot reflect the quantitative characteristics and image characteristics of the subway network. In this study, the hypergraph space structure of the Nanjing subway network is used for research. In this study, the space-time evolution mechanism of vulnerability of the Nanjing subway system is extended. The hypergraph space structure is quantitatively and qualitatively analyzed by establishing superedge degree, node superdegree, and superedge weight indexes, and is compared with the indexes of network space and space $P$ structure. Different from previous studies by scholars, previous analysis of subway spatial vulnerability focuses on static analysis, while this study uses the method of time-space evolution mechanism to dynamically and quantitatively analyze the gradual change characteristics of Nanjing subway system vulnerability under the influence of time and space. In addition, this study also makes static and dynamic comparative analysis on the vulnerability of the subway system by using connectivity rate and trip efficiency index, which provides new analysis methods and ideas for the study of the subway system network.

Compared with network space and space $P$, the node superdegree of hypergraph space structure shows more uneven distribution. Nanjing's subway network space, space $P$, and hypergraph space have the same size, which indicates that the size of subway space has a linear relationship with the opening of subway lines.

Through the static analysis of the vulnerability of the Nanjing subway system, it is found that in the hypergraph space of the subway system, the transfer stations and lines with higher node superdegree, superedge degree, and superedge weight have higher vulnerability performance and should be listed as the key protection areas of the subway. When no more than $50 \%$ of Nanjing subway stations and lines are attacked, the entire subway system will basically lose its function. Therefore, in the subsequent subway construction, it is necessary to adjust the planning and increase the construction of the subway loop line, so as to improve the tightness of the connection between the suburban line and the main city line.

Compared with previous researches by scholars, the biggest advantage of Nanjing subway vulnerability spacetime evolution analysis is that fully takes into account the changes of results brought by the changes of time and space variables.

According to the analysis of the space-time evolution of Nanjing Metro vulnerability, the overall network structure of Nanjing Metro shows a loose trend. After the completion of the connecting line between the main city and the suburbs, Nanjing Metro will focus on the construction of the ring line. Therefore, the vulnerability of the metro system should be reduced and the reliability will be improved.

The Nanjing subway line not only formed the complex network space structure of Nanjing subway but also changed the planning pattern and traffic development of Nanjing city. According to the plan issued by official website of Nanjing Metro before the end of 2020, 12 new subway lines will be built. So, Nanjing subway lines will form a more complicated space structure, which needs further research and discovery.

\section{Data Availability}

The data used to support the findings of this study have been deposited in the 4TU.ResearchData repository (DOI: 10.4121/uuid:a0492dbc-2e8c-4577-93c0-867d4521fcdd), are included within the article, were supplied by Xiaoyan Gu under license and so cannot be made freely available (requests for access to these data should be made to Xiaoyan Gu, gxy@jit.edu.cn), are currently under embargo while the research findings are commercialized (requests for data, 12 months after publication of this article, will be considered by the corresponding author), may be released upon application to the (4TU.ResearchData), who can be contacted at (https://data.4tu.nl/), and are available from the corresponding author upon request.

\section{Conflicts of Interest}

The authors declare no conflicts of interest.

\section{Acknowledgments}

This work was supported by the Project of Humanities and Social Sciences Research and Planning Fund of the Ministry of Education (project no.19YJA790016), Supported Projects of Jiangsu Blue and Green Project, and Special Key Projects of Jiangsu Social Science Fund Series (17DDA003).

\section{References}

[1] B. Ye, Research on the Cause Analysis and Risk Prevention and Control Measures of Nanjing Metro Operation Fault Based on ISM Method, Southeast University, Nanjing, China, 2018.

[2] C. Pozrikidis, "Node degree distribution in spanning trees," Journal of Physics A Mathematical \& Theoretical, vol. 49, no. 12, Article ID 125101, 2016. 
[3] L. R. Gorjão, A. Saha, G. Ansmann, U. Feudel, and K. Lehnertz, "Complexity and irreducibility of dynamics on networks of networks," Nonlinear Sciences, vol. 28, no. 10, 2018.

[4] G. Alexander, L. Samokhine, and P. Bocharov, "About complexity of complex networks," Applied Network Science, vol. 4, no. 1, 2019.

[5] S. An, L. Ma, and J. Wang, "Optimization of traffic detector layout based on complex network theory," Sustainability, vol. 12 , no. 5 , p. $2048,2020$.

[6] S. Karunakaran and L. Selvaganesh, "A novel graph matrix representation: sequence of neighbourhood matrices with an application," SN Applied Sciences, vol. 2, no. 5, 2020.

[7] L. Beranek and R. Remes, "Distribution of node characteristics in evolving tripartite network," Entropy, vol. 22, no. 3, p. $263,2020$.

[8] D. N. A. Mensah, H. Gao, and L. Yang, "Approximation algorithm for shortest path in large social networks," Algorithms, vol. 13, no. 2, p. 36, 2020.

[9] D. Džamić, J. Pei, M. Marić, N. Mladenović, and P. Pardalos, "Exponential quality function for community detection in complex networks," International Transactions in Operational Research, vol. 27, no. 1, pp. 245-266, 2018.

[10] V. Latora and M. Marchiori, "Is the Boston subway a smallworld network?," Physica A: Statistical Mechanics \& Its Applications, vol. 314, no. 1-4, pp. 109-113, 2002.

[11] A. Mrvar, V. Batagelj, and W. de Nooy, Exploratory Social Network Analysis with Pajek, Cambridge University Press, Cambridge, UK, 2011.

[12] F. Ma, H. Xue, K. F. Yuen et al., "Assessing the vulnerability of logistics service supply chain based on complex network," Sustainability, vol. 12, 2020.

[13] H. Abdelaty, M. Ezzeldin, E. Mohamed, and W. ElDakhakhni, "Quantifying and classifying the robustness of bus transit networks," Transportmetrica A: Transport Science, vol. 16, no. 3, pp. 1176-1216, 2020.

[14] O. D. Polishchuk, "Vulnerability of complex network structures and systems," Cybernetics and Systems Analysis, vol. 56, no. 2, pp. 312-321, 2020.

[15] D. J. Sun, Y. Zhao, and Q.-C. Lu, "Vulnerability analysis of urban rail transit networks: a case study of Shanghai, China," Sustainability, vol. 7, no. 6, pp. 6919-6936, 2015.

[16] S. Derrible and C. Kennedy, "The complexity and robustness of metro networks," Statistical Mechanics and Its Applications, vol. 389, no. 17, pp. 678-691, 2010.

[17] G. G. Malinetskii and M. E. Stepantsov, "A discrete mathematical model of the dynamic evolution of a transportation network," Computational Mathematics \& Mathematical, vol. 49, pp. 1493-1498, 2009.

[18] Y. J. Kim, D. N. Mavris, and R. Fujimoto, "Time- and spaceparallel simulation of air traffic networks," Simulation, vol. 95, no. 12, 2019.

[19] M. K. Hosseini and A. Talebpour, "Traffic prediction using time-space diagram: a convolutional neural network approach," Transportation Research Record: Journal of the Transportation Research Board, vol. 2673, no. 7, pp. 425-435, 2019.

[20] F. Su, H. Dong, L.-M. Jia, and T. Zhao, "Space-time correlation analysis of traffic flow on road network," International Journal of Modern Physics B, vol. 31, no. 5, 2016.

[21] S. D. Bačkalić, B. Matović, and A. Bašić, "The time-space approach in the analysis of traffic safety on rural road," Romanian Journal of Transport Infrastructure, vol. 6, no. 1, pp. 66-76, 2017.
[22] Z. Qu and S. He, "A time-space network model based on a train diagram for predicting and controlling the traffic congestion in a station caused by an emergency," Symmetry, vol. 11, no. 6, 2019.

[23] Y. Kang, N. Cho, and S. Son, "Spatiotemporal characteristics of elderly population's traffic accidents in Seoul using spacetime cube and space-time kernel density estimation," PLoS One, vol. 13, no. 5, Article ID e0196845, 2018.

[24] D. J. Zawack and G. L. Thompson, "A dynamic space-time network flow model for city traffic congestion," Transportation Science, vol. 21, no. 3, pp. 153-162, 1987.

[25] D. Sun and S. Guan, "Measuring vulnerability of urban metro network from line operation perspective," Transportation Research Part A: Policy \& Practice, vol. 94, pp. 48-359, 2016.

[26] X. Ding, S. Guan, and D. J. Sun, "Short turning pattern for relieving metro congestion during peak hours," European Transport Research Review, vol. 10, no. 28, 2018.

[27] G. Nian, F. Chen, Z. Li, Y. Zhu, and D. J. Sun, "Evaluating the alignment of new metro line considering network vulnerability with passenger ridership," Transportmetrica A: Transport Science, vol. 15, no. 2, pp. 1402-1418, 2019.

[28] P. Crucitti, V. Latora, M. Marchiori, and A. Rapisarda, "Efficiency of scale-free networks: error and attack tolerance," Physica A: Statistical Mechanics and its Applications, vol. 320, pp. 622-642, 2003.

[29] Z. Su, L. Li, H. Peng, J. Kurths, J. Xiao, and Y. Yang, "Robustness of interrelated traffic networks to cascading failures," Scientific Reports, vol. 4, p. 5413, 2014.

[30] A. D. Broido and A. Clauset, "Scale-free networks are rare," Nature Communications, vol. 10, 2019. 